\title{
Endoscopic ultrasonography-guided antegrade stenting combined with hepatico- gastrostomy/hepaticojejunostomy using ultraslim instruments
}

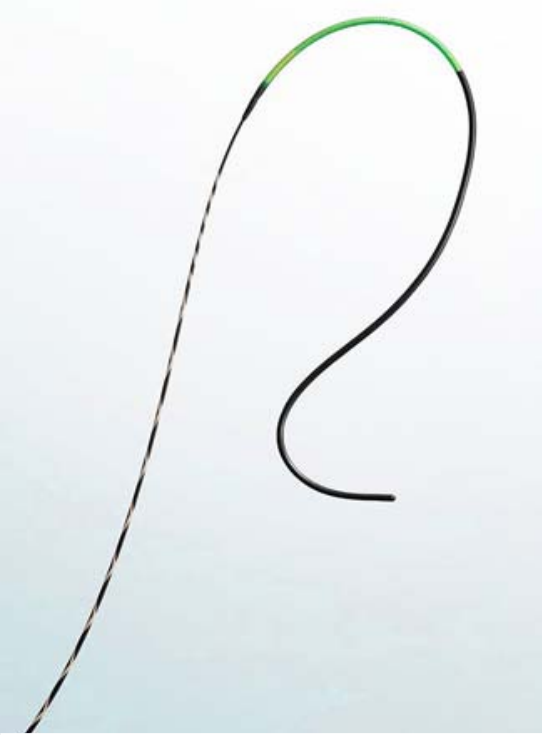

- Fig. 1 The VisiGlide 2 (0.025 inch, angled type; Olympus, Tokyo, Japan) has enhanced tip flexibility and provides the same stiffness as a conventional 0.035 -inch guidewire.

Techniques for endoscopic ultrasonography (EUS)-guided biliary drainage (EUSBD) have been developed, and EUS-guided antegrade stenting (EUS-AGS) and EUS-guided hepaticogastrostomy (EUS-
HGS)/hepaticojejunostomy (HJS) are suitable for gastric outlet obstruction (GOO) or surgically altered anatomy. EUS-AGS alone carries the potential risk of causing bile leakage from a fistula; however, EUS-AGS in combination with EUS-HGS or EUS-HJS appears safer, as it can reduce the risk of a bile leak [1,2]. We present two patients who underwent EUS-HGS or EUS-HJS combined with EUSAGS using ultraslim instruments. Patient \#1 was a 62-year-old woman who had undergone a previous total gastrectomy for gastric cancer and later developed obstructive jaundice. First, a B3 branch was punctured using a 196 needle via a transjejunal approach, and a 0.025-inch guidewire (VisiGlide 2; Olympus, Tokyo, Japan) (\Fig. 1) was placed. Next, a tapered endoscopic retrograde cholangiopancreatography (ERCP) catheter (01 2021 1; MTW Endoskopie, Düsseldorf, Germany) ( $\triangleright$ Fig.2) was used to dilate the fistula, following successful passage of the guidewire through the stricture. EUS-AGS was then performed using a novel ultraslim uncovered self-expandable metal stent (SEMS; BileRush Selective; 5.7 Fr, 10-mm diameter; Piolax Medical Devices, Kanagawa, Japan)

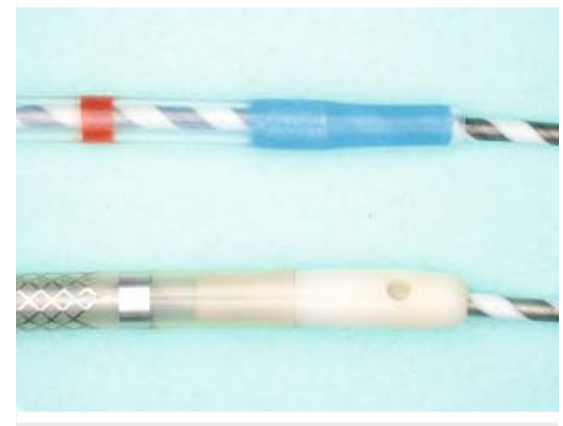

- Fig. 2 A tapered endoscopic retrograde cholangiopancreatography catheter (01 2021 1; 4.8-Fr tip diameter, 6.9-Fr shaft diameter; MTW Endoskopie, Düsseldorf, Germany) passed over a 0.025 -inch guidewire (upper image) and a novel ultraslim uncovered self-expandable metal stent (BileRush Selective; $5.7 \mathrm{Fr}, 10$-mm diameter, 185-cm long; Piolax Medical Devices, Kanagawa, Japan) (lower image).

( Fig.2). Finally, a novel 7-Fr plastic stent (TYPE-IT stent; Gadelius Medical Co. Ltd., Tokyo, Japan) [3] (> Fig.3) was placed to create an EUS-HJS ( $>$ Fig. 4; - Video 1).

Patient \#2 was a 68-year-old man with GOO caused by gastric cancer who developed obstructive jaundice. EUS-AGS and EUS-HGS were performed as described
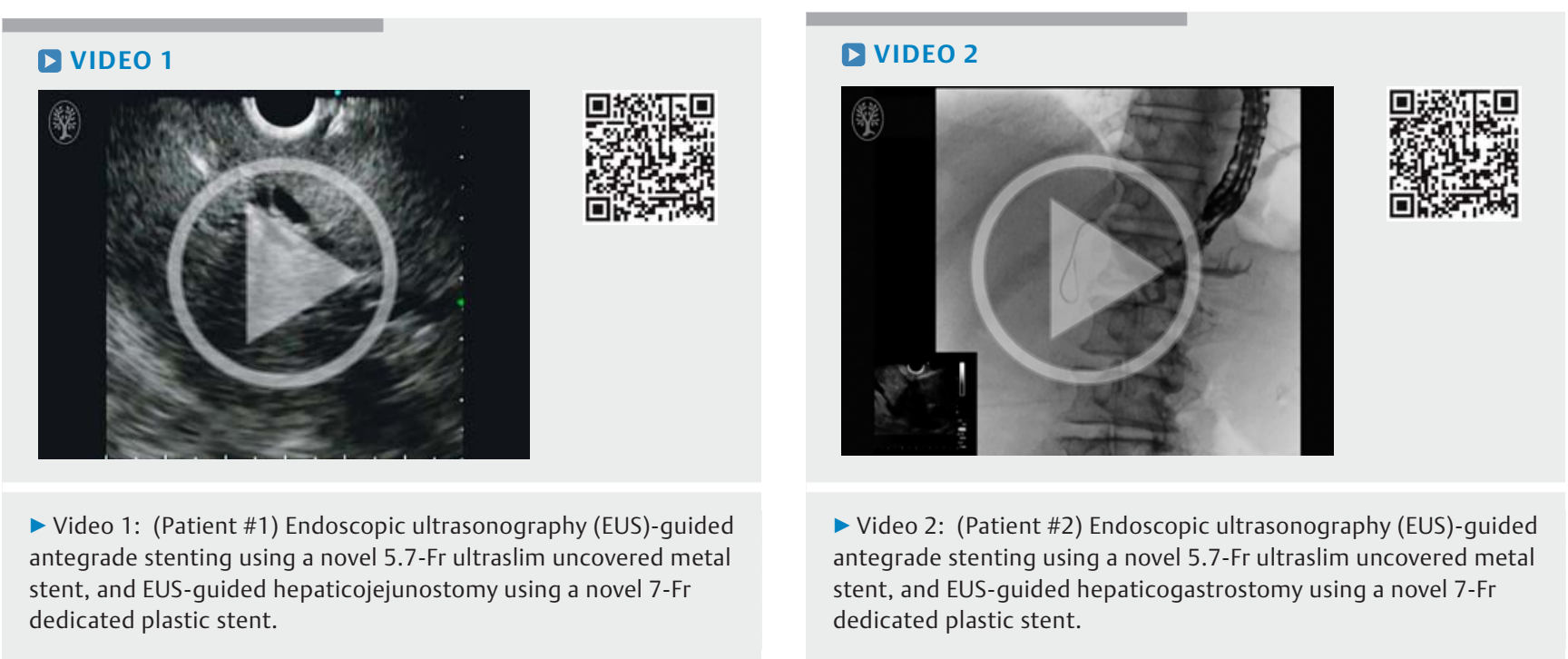


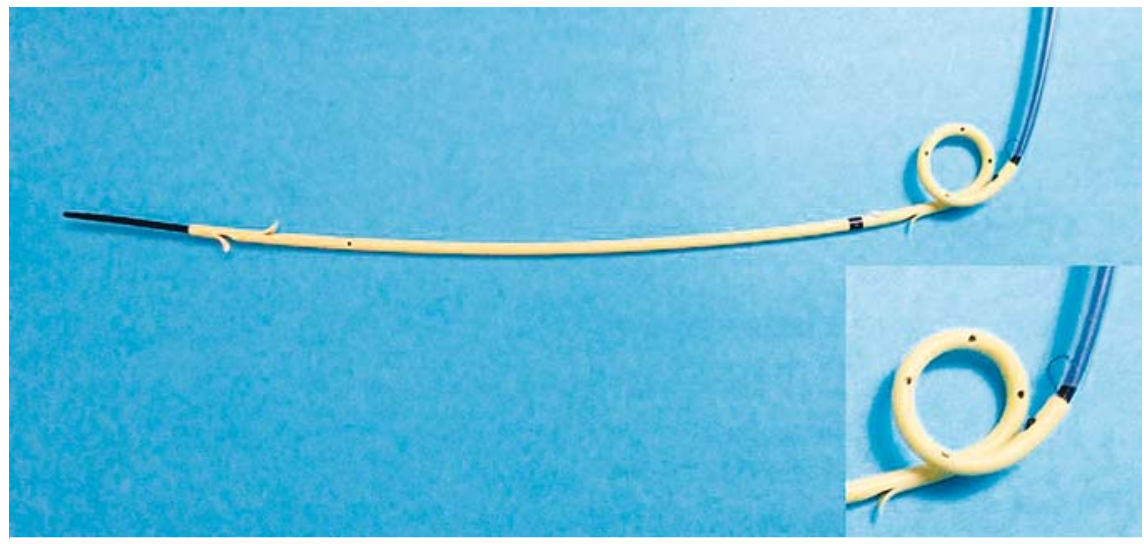

- Fig. 3 A novel plastic stent (TYPE-IT stent; Gadelius Medical Co. Ltd., Tokyo, Japan) can be retracted with a lasso, and has a $7-\mathrm{Fr}$ diameter (5-Fr inner catheter-tip diameter), with total length of $20 \mathrm{~cm}$, effective length of $15 \mathrm{~cm}$, single-pigtail type (proximal end), and four flanges (two at the distal end and two at the proximal end).

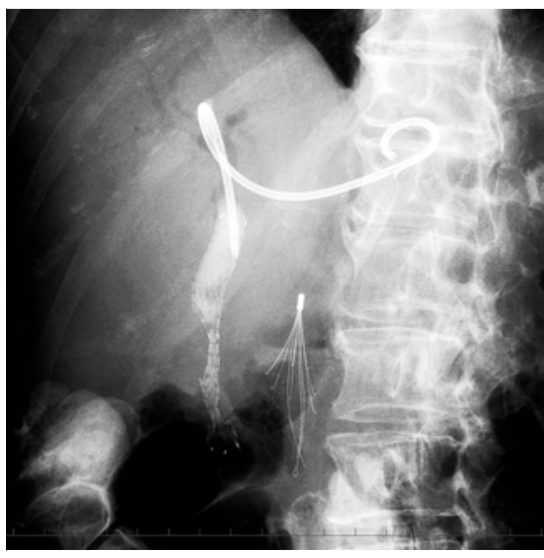

- Fig. 4 (Patient \#1) Radiograph showing an endoscopic ultrasonography-guided hepaticojejunostomy with a dedicated $7-\mathrm{Fr}$ plastic stent and antegrade stenting with a 5.7-Fr uncovered metal stent.

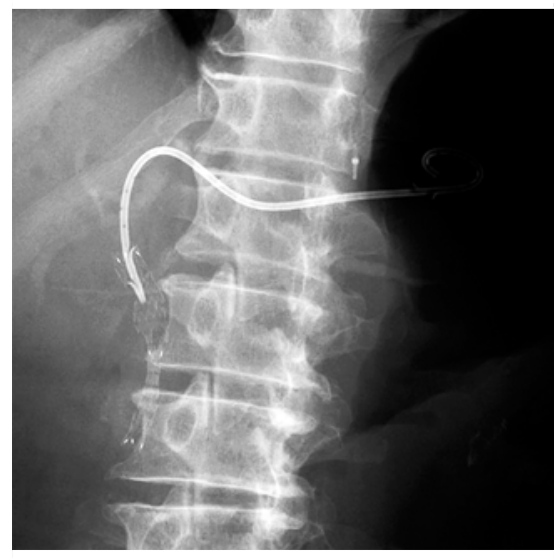

- Fig. 5 (Patient \#2) Radiograph showing an endoscopic ultrasonography-guided hepaticogastrostomy with a dedicated 7-Fr plastic stent and antegrade stenting with a 5.7-Fr uncovered metal stent.

can facilitate the procedure and minimize the potential for bile leakage.

no complications in either case.

A covered SEMS (CSEMS) is commonly used to prevent bile leaks in EUS-HGS/ HJS. A long partially covered SEMS (PCSEMS; $\geq 10 \mathrm{~mm}$ ) can be used to prevent stent migration [4]. However, the thicker delivery system $(8.5 \mathrm{Fr})$ with this long PCSEMS and the cost of two metal stents are of concern. In particular, minimum fistula dilation should be performed during EUS-BD. Therefore, EUS-AGS and EUS-HGS/HJS using various ultraslim instruments (7 Fr or less) in combination

\section{Competing interests}

A novel ultraslim uncovered metal $10-\mathrm{mm}$ diameter, $185-\mathrm{cm}$ long) has been developed through collaborative research between Dr. Kawakami and Piolax Medical Devices, Kanaga- wa, Japan. Dr. Kawakami is a consultant and gives lectures for the Piolax Medical Devices and for Olympus, Tokyo, Japan. Dr. Kubota has no competing interests to declare.

\section{The Authors}

Hiroshi Kawakami, Yoshimasa Kubota

Department of Gastroenterology and

Hepatology, Faculty of Medicine, University of Miyazaki and Center for Digestive Disease, University of Miyazaki Hospital, Miyazaki, Japan

\section{Corresponding author}

\section{Hiroshi Kawakami, MD, PhD}

Department of Gastroenterology and Hepatology, Faculty of Medicine, University of Miyazaki, Center for Digestive Disease, University of Miyazaki Hospital, 5200, Kihara, Kiyotake, Miyazaki 889-1692, Japan Fax: +81-985-859802

hiropon@med.miyazaki-u.ac.jp

\section{References}

[1] Ogura T, Masuda D, Imoto A et al. EUSguided hepaticogastrostomy combined with fine-gauge antegrade stenting: a pilot study. Endoscopy 2014; 46: 416-421

[2] Kumbhari V, Tieu AH, Khashab MA. EUSguided biliary drainage made safer by a combination of hepaticogastrostomy and antegrade transpapillary stenting. Gastrointest Endosc 2014; 81: 1015 - 1016

[3] Umeda J, Itoi T, Tsuchiya T et al. A newly designed plastic stent for EUS-guided hepaticogastrostomy: a prospective preliminary feasibility study (with videos). Gastrointest Endosc 2015; 82: 390 -396.e2

[4] Nakai $\mathrm{Y}$, Isayama $\mathrm{H}$, Yamamoto $\mathrm{N}$ et al. Safety and effectiveness of a long, partially covered metal stent for endoscopic ultrasound-guided hepaticogastrostomy in patients with malignant biliary obstruction. Endoscopy 2016; 48: 1125-1128 stent (BileRush Selective; 5.7 Fr, 8-mm/

\section{Bibliography}

DOI http://dx.doi.org/10.1055/s-0043-101225

Endoscopy 2017; 49: E88-E89

(c) Georg Thieme Verlag KG

Stuttgart - New York

ISSN 0013-726X 